\title{
Spherical Particles in As-prepared Al65Cu25Fe15 Alloy by Arc-melting
}

\author{
Jeremy Marshall, Heavenly Duley, Joshua Homan, Clay Klein and Chunfei Li \\ Clarion University of Pennsylvania, Clarion, Pennsylvania, United States
}

$\mathrm{Al}_{65} \mathrm{Cu}_{25} \mathrm{Fe}_{15}$ alloy is well known in the field of quasicrystalline material, mainly because of the early reports that it is suitable for preparing pure icosahedral quasicrystalline (IQC) phase [1]. However, followup studies have revealed a far more complicated picture. The alloy prepared by arc-melting appears to be porous, consisting of multiple voids. On the surface of such voids, there are three types of particles with distinct morphologies. These particles resemble slab, sphere, and pentagonal dodecahedron. The present report concerns the spherical particles. It has been assumed that the spherical particles are all of the same phase but different grains. Our recent examination has revealed that the spherical particles tend to line-up along the length direction of the slab-shaped $\lambda$ phase. The surface is decorated with facets, which are arranged in similar patterns on adjacent particles [2]. It has been speculated that although the adjacent spherical particles appear to be independent, they might be part of a single crystal.

There are two possible approaches to test this hypothesis. First, Transmission Electron Microscope (TEM) can be used. In doing so, specimen preparation presents a serious challenge and the specimen handling could introduce significant uncertainty. On the other hand, Electron Back Scattering Diffraction (EBSD) is a technique based on Scanning Electron Microscope (SEM). EBSD patterns can be taken from a very large area. When such patterns are used to analyze the orientation relationship between different particles, it is expected that there is less uncertainty due to instrument operation and specimen handling. The concern with EBSD is whether good EBSD patterns can be obtained, especially for the present case when the sample surface is very rough. The result of our attempt to study the orientation relationship between such spherical particles based on the usage of EBSD is reported here.

The as-prepared $\mathrm{Al}_{65} \mathrm{Cu}_{25} \mathrm{Fe}_{15}$ alloy by arc-melting was mechanically crushed to small pieces to expose the porous surface, where spherical particles are located. The obtained small pieces are mounted on an SEM stub with double-sided carbon tape. An extensive survey was carried out to locate pieces that are suitable for the present study. Effort is made to select pieces that are small and stand on relatively high ground. This way, EBSD signals can be collected despite the surface roughness over a large range. Fig. 1(a) shows the SEM image of such a piece, where the letters on the image show the locations of spherical particles where EBSD patterns have been taken. Figs. 1(b)-(e) show the corresponding EBSD patterns. Despite the fact that spherical particles appear to be independent particles, the EBSD patterns resemble strongly with each other, proving our speculation that different spherical particles are parts of a single crystal. This result demonstrates the effectiveness of EBSD in examining orientation relationship. A model to explain the individual particle appearance and the underlying single crystal nature will be suggested. The possible relationship of the spherical particles with icosahedral quasicrystalline phase inside it and the $\lambda$ phase beneath it will be investigated as well. 

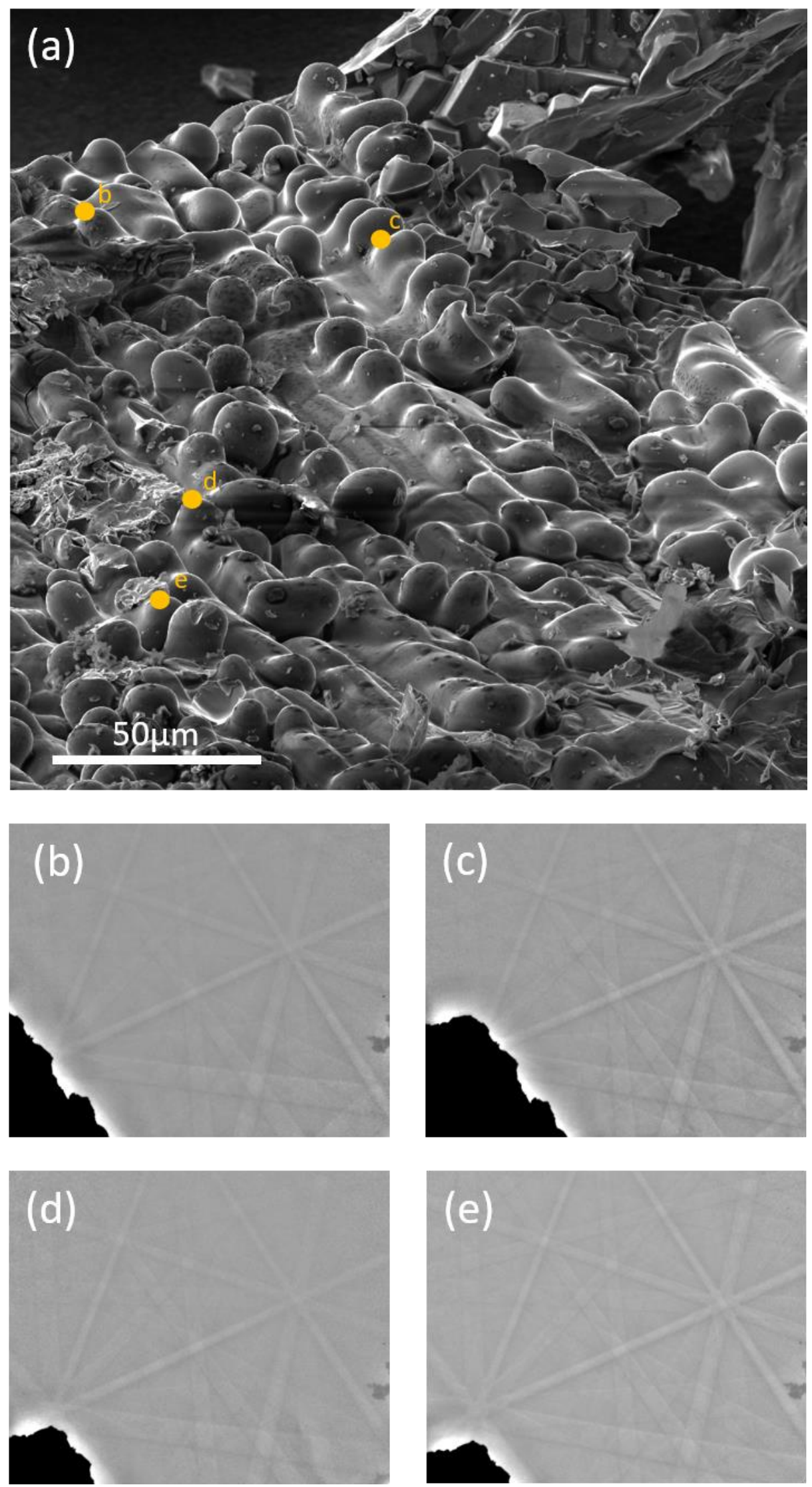
Figure 1. SEM image (a) of multiple spherical particles and EBSD patterns (b) to (e) from corresponding spherical particles marked on the SEM image. Resemblance of EBSD patterns imply that particles are parts of a single crystal.

\section{References}

[1]. Balzuweit, K., Meekes, H., Tendeloo, G. V., \& De Boer, J. L. (1993). On the relationship between morphology, composition and structure of Al-Cu-Fe crystals and quasicrystals. Philosophical Magazine $\mathrm{B}, 67(4), 513-532$.

[2]. Li, C., Kothe, B., Caputo, M., \& Hampikian, H. (2017). Ordering of Nanoparticles along Concentric Nanoings Observed in Al-Cu-Fe Alloy. Microscopy and Microanalysis, 23(S1), 1960-1961. 